\title{
CHEMOTAXIS UNDER AGAROSE UTILIZING HUMAN SERUM DEPLETED OF C-5 DERIVED PEPTIDES ${ }^{1}$
}

\author{
GEORGE L. MANDERINO *, ADORACION F. SUAREZ *, ARTHUR A. HIRATA * \\ and PETER A. WARD ** \\ * Laboratory of Immunology, Abbott Laboratories, North Chicago, IL 60064, and \\ ** Department of Pathology, University of Michigan Medical School, Ann Arbor, MI \\ 48109, U.S.A.
}

(Received 21 April 1981, accepted 13 May 1981)

An immunoabsorbent column was made with antibody to trypsinized human C5. This column removed the chemotactic activity from zymosan-activated serum as well as from C5a des-arg-enriched fractions. Anti-trypsinized C5-absorbed human serum was substituted for unabsorbed human serum in the chemotaxis under agarose system. This resulted in significantly reduced random neutrophil migration with a negligible effect on C5a des-arg or FMLP-directed migration. The results indicate that much of the random migration observed in the chemotaxis under agarose system is due to C5-derived peptides present in normal human serum.

\section{INTRODUCTION}

Leukocytes from several species migrate under agarose in either a random or directed manner, the latter response being to a chemotactic gradient. Since its description by Cutler (1974), multiple variations of this technique have been described (Nelson et al., 1975; Repo, 1977; Chenoweth et al., 1979; Rabinovitch et al., 1980; Tannous and Cavender, 1980). The major advantages of the migration under agarose method include: simplicity, requirement of small leukocyte numbers and microliter volumes of reagents, and the ability to assess both random and directed migration in the same population of cells.

The agarose chemotaxis system requires the presence of serum or albumin (Cutler, 1974; Nelson et al., 1975; Repo, 1977) or gelatin (Chenoweth et al., 1979). Although the use of serum in agarose provides a more physiological milieu, serum is known to contain both cytotaxins and inhibitors of leuko-

\footnotetext{
${ }^{1}$ Supported in part by NIH Grants AICA 17334 and GM 28499-01.

Abbreviations: FMLP, N-formyl-L-methionyl-L-leucyl-L-phenylalanine; E-MEM, Minimum Essential Medium with Earle's salts; HINHS, heat-inactivated normal human AB serum; HIZAS, heat-inactivated zymosan-activated normal human AB serum; S-anti-tryp-C5, Sepharose 6B-anti-trypsinized C5; CI, chemotactic index.
} 
cyte migration. In addition, various serum pools might contain different quantities of these effectors which can lead to a lack of reproducibility among different serum pools (Nelson et al., 1975).

Coagulation has long been recognized as a means of generating cytotaxins (Kaplan et al., 1972). Wiggins and Giclas (1980) have recently reported that the incubation of highly purified rabbit kallikrein with complement component C5 generated chemotactic peptides. The data presented in this paper demonstrate that absorption of heat-inactivated normal human serum with Sepharose-anti-trypsinized C5 prior to its incorporation into agarose significantly reduced random leukocyte migration without significantly affecting C5a des-arg- or FMLP-directed migration. Immunoabsorption of serum with antibody to $\mathrm{C} 5$ chemotactic peptides results in increased chemotactic indices as well as greater reproducibility among different serum pools.

\section{MATERIALS AND METHODS}

\section{Preparation of human leukocytes}

Blood from healthy volunteers was drawn into disposable syringes containing $100 \mathrm{U}$ of preservative-free sodium heparin $/ \mathrm{ml}$ of blood. Erythrocytes were sedimented by gravity following the addition of $1 \mathrm{ml}$ of $6 \%$ Dextran T 70 (Pharmacia Fine Chemicals, Piscataway, NJ) (w/v) in physiological saline to $3 \mathrm{ml}$ of blood. The leukocyte-rich plasma was centrifuged at $150 \times \mathrm{g}$ for $10 \mathrm{~min}$, the cells washed twice in E-MEM (Grand Island Biological Co., Grand Island, NY) and residual erythrocytes removed by hypotonic lysis. The leukocyte suspension routinely contained $50-85 \%$ neutrophils and was $>97 \%$ viable as determined with acridine orange, ethidium bromide fluorescence (Lee et al., 1975). Leukocytes were suspended in E-MEM supplemented with $100 \mathrm{U} / \mathrm{ml}$ penicillin, $100 \mu \mathrm{g} / \mathrm{ml}$ streptomycin, $20 \mathrm{mM}$ HEPES buffer, $2.2 \mathrm{mg} / \mathrm{ml} \mathrm{NaHCO}$ to contain $2.5 \times 10^{7}$ neutrophils $/ \mathrm{ml}$.

\section{Preparation of serum}

Blood from $\mathrm{AB}$ donors was allowed to clot at $37^{\circ} \mathrm{C}$ for $30 \mathrm{~min}$ followed by $90 \mathrm{~min}$ at $4^{\circ} \mathrm{C}$. Serum was collected after centrifugation at $800 \times g$ for 30 min and lipids removed by ultracentrifugation $(100,000 \times g)$ for $1 \mathrm{~h}$. Following heat inactivation $\left(56^{\circ} \mathrm{C}\right.$ for $\left.30 \mathrm{~min}\right)$ the serum, designated HINHS, was passed through $0.45 \mu \mathrm{m}$ filters (Nalge Co., Rochester, NY) and stored in small aliquots at $-80^{\circ} \mathrm{C}$ until used.

Alternately, AB plasma was obtained from a local blood bank and converted into serum by adding $\mathrm{CaCl}_{2}$ to $10 \mathrm{mM}$ and $2 \mathrm{U} / \mathrm{ml}$ thrombin (ParkeDavis, Moris Plains, NJ) and incubated for $18 \mathrm{~h}$ at $4^{\circ} \mathrm{C}$. Serum was freed of clots by passage through cheesecloth and ultracentrifuged at $100,000 \times g$ to remove lipids. The serum was heat-inactivated, filtered and stored as described above.

Absorption of serum with S-anti-tryp-C5

Complement component C5 was digested with trypsin as previously 
described (Ward and Newman, 1969). Antibodies to trypsinized C5 were raised in goats and the serum IgG was isolated according to the method of Gray et al. (1969). Reactivity of IgG anti-trypsinized C5 with highly purified C5a isolated, as described by Vallota and Müller-Eberhard (1973), was demonstrated by immunodiffusion.

Five $\mathrm{mg} / \mathrm{ml}$ of the IgG fraction of anti-trypsinized C5 were coupled to Sepharose 6B (Pharmacia Fine Chemicals) following cyanogen bromide activation (Cuatrecasas et al., 1968) and is referred to as S-anti-tryp-C5. Two volumes of HINHS were incubated with 0.5 vol of packed S-anti-tryp-C5 for $30 \mathrm{~min}$ at $4^{\circ} \mathrm{C}$ after which the latter was removed by centrifugation $(400 \times \mathrm{g}$ for $10 \mathrm{~min}$ ). The S-anti-tryp-C5-absorbed serum was stored at $-80^{\circ} \mathrm{C}$ until used. Alternately, HINHS was passed over a column containing S-anti-trypC5 with the same results. As a control, HINHS was absorbed with unconjugated Sepharose 6B.

\section{Preparation of agarose plates}

Agarose plates for chemotaxis were made as described by Nelson et al. (1975) with minor modifications. In brief, a $5 \mathrm{ml}$ mixture of $1 \%$ agarose (Seakem, Marine Colloids, Rockland, ME) in supplemented E-MEM containing $10 \%$ HINHS or $10 \%$ S-anti-tryp-C5-absorbed HINHS was added to each $60 \mathrm{~mm} \times 15 \mathrm{~mm}$ tissue culture dish (Falcon, Oxnard, CA). Six series of 3 wells $3 \mathrm{~mm}$ in diameter and spaced $3 \mathrm{~mm}$ apart were cut in each plate with a plexiglass template and stainless steel punch.

\section{Chemotactic factors}

Fresh human $A B$ serum was activated with $10 \mathrm{mg} / \mathrm{ml}$ zymosan (Sigma Chemical Co., St. Louis, MO) for $60 \mathrm{~min}$ at $37^{\circ} \mathrm{C}$. The zymosan was removed by centrifugation and the serum inactivated for $30 \mathrm{~min}$ at $56^{\circ} \mathrm{C}$. The resulting HIZAS was passed over S-anti-tryp-C5 columns and a C5a des-arg-enriched fraction was eluted with $0.2 \mathrm{M}$ glycine $\mathrm{HCl}$ buffered saline $\mathrm{pH}$ 2.8. Following dialysis vs. PBS, the C5a des-arg-enriched fraction was passed over Sephadex G-75 (Pharmacia Fine Chemicals) and the resultant fractions were assayed in the agarose chemotaxis system for chemotactic activity. Active fractions, found only in the 10,000-15,000 Mr range, were pooled and titrated for chemotactic activity. A dilution in E-MEM giving optimal activity was subsequently used in chemotaxis assays. N-formyl-L-methionyl-L-leucyl-Lphenylalanine (FMLP) was purchased from Vega Biochemicals (Tucson, AZ). Stock solutions, $10^{-3} \mathrm{M}$ in DMSO, were diluted to $10^{-7} \mathrm{M}$ in supplemented E-MEM for chemotaxis assays.

\section{Chemotaxis assays}

Ten microliters of chemotactic factor were added to the outer wells, $10 \mu \mathrm{l}$ of E-MEM to the inner wells, and $10 \mu \mathrm{l}$ of leukocyte suspensions containing $2.5 \times 10^{5}$ neutrophils to the central wells of agarose plates. After incubating at $37^{\circ} \mathrm{C}$ for various lengths of time in humidified $5 \% \mathrm{CO}_{2} / 95 \%$ 
air, the cells were fixed, stained, and their migration quantitated as described by Nelson et al. (1975). Chemotactic indices were determined by calculating the ratio between the mean migration distances toward either C5a des-arg or FMLP and those toward E-MEM (Nelson et al., 1975).

\section{RESULTS}

\section{Abrogation of C5a des-arg-directed migration by S-anti-tryp-C5}

The ability of S-anti-tryp-C5 to absorb chemotactic complement factors from human serum was assessed. The chemoattractants HIZAS and C5a desarg were absorbed with either S-anti-tryp-C5 or control Sepharose prior to their addition to the outer wells of $1 \%$ agarose plates containing $10 \%$ HINHS simultaneously with the application of neutrophils to the central wells. The plates were incubated at $37^{\circ} \mathrm{C}$ for $2 \mathrm{~h}$, the cells fixed and stained, and migration distances measured. As shown on Table 1, absorption of HIZAS or C5a des-arg with unconjugated Sepharose had no effect on their chemotactic properties. After absorption with S-anti-tryp-C5, however, neither HIZAS nor C5a des-arg was capable of inducing neutrophil migration farther than that elicited by random migration toward E-MEM or HINHS.

\section{Effect of preabsorbing HINHS with S-anti-tryp-C5 on random neutrophil migration}

Random migration of human neutrophils was assessed in $1 \%$ agarose containing either $10 \%$ HINHS or 10\% S-anti-tryp-C5-absorbed HINHS. E-MEM was added to the inner and outer wells simultaneously with the application of leukocytes containing $2.5 \times 10^{5}$ neutrophils to the central wells of the

\section{TABLE 1}

Abrogation of C5a des-arg chemotactic activity by Sepharose-anti-trypsinized C5 affinity columns ${ }^{\text {a }}$.

\section{Attractant}

Migration $(\mu \mathrm{m})$

(mean \pm S.D.

of triplicate samples)

\section{HINHS}

$580 \pm 45$

HIZAS

$1615 \pm 155$

HIZAS passed over Sepharose-control columns

$1580 \pm 125$

HIZAS passed over Sepharose-anti-trypsinized C5 columns

$530 \pm 50$

E-MEM

C5a des-arg

C5a des-arg passed over Sepharose-control columns

C5a des-arg passed over Sepharose-anti-trypsinized C5 columns

$520 \pm 80$

$1710 \pm 190$

$1680 \pm 115$

$530 \pm 35$

a Ten microliters of attractant were added to the outer wells of $1 \%$ agarose plates containing $10 \%$ HINHS simultaneously with the application of $2.5 \times 10^{5}$ neutrophils to the central wells. Migration distances were measured after a $2 \mathrm{~h}$ incubation at $37^{\circ} \mathrm{C}$. 
agarose plates. After incubating at $37^{\circ} \mathrm{C}$ for various lengths of time, the cells were fixed, stained, and their migration distances were measured. As a representative experiment depicted in Fig. 1 demonstrates, random migration distances increased with time in both HINHS- and S-anti-tryp-C5-

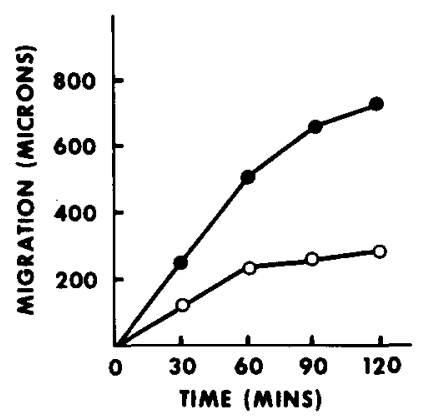

Fig. 1. Time-dependent random neutrophil migration under agarose. E-MEM was added to the inner and outer wells simultaneously with the application of leukocytes containing $2.5 \times 10^{5}$ neutrophils to the central wells of $1 \%$ agarose containing $10 \%$ HINHS $(\bullet-)$ or $10 \%$ S-anti-tryp-C5-absorbed HINHS $(\circ-0)$. Each point represents the mean value obtained from quadruplicate determinations with S.D. $<10 \%$ from a representative experiment.

absorbed HINHS-containing plates. At all time intervals examined, however, random neutrophil migration in the latter was consistently less than half that observed in the former. Characteristic migration patterns achieved after $2 \mathrm{~h}$ of incubation are shown in Fig. 2.

Effect of preabsorbing HINHS with S-anti-tryp-C5 on C5a des-arg or FMLPdirected neutrophil migration

In the next series of experiments, C5a des-arg or FMLP was added to the outer wells, E-MEM to the inner wells and $2.5 \times 10^{5}$ neutrophils to the central wells of $1 \%$ agarose plates containing $10 \%$ HINHS or $10 \%$ S-anti-tryp-C5absorbed HINHS. Fig. 3A depicts the migration distances toward C5a des-arg when this reagent was added simultaneously with the application of leukocytes to the above plates. In both systems, migration to C5a des-arg after 30 min incubation was no greater than random migration distances (compare with Fig. 1). During the next $90 \mathrm{~min}$, however, migration to C5a des-arg increased almost linearly with time in both HINHS- and S-anti-tryp-C5absorbed HINHS-containing agarose. At all time intervals examined, greater migration distances were observed in the agarose-containing HINHS. When FMLP was substituted for C5a des-arg in the above experiments, directed migration was observed as early as $\mathbf{3 0}$ min after the application of leukocytes in both HINHS- and S-anti-tryp-C5-absorbed HINHS-containing agarose (Fig. 3B). At each time interval almost identical migration distances were attained in both agarose systems. 
The chemotactic response of neutrophils under agarose in related to a concentration gradient that is established by radial diffusion of the stimulus factor from the site of application (Nelson et al., 1975; Repo, 1977). Cells migrating spontaneously toward the well containing the chemotactic factor

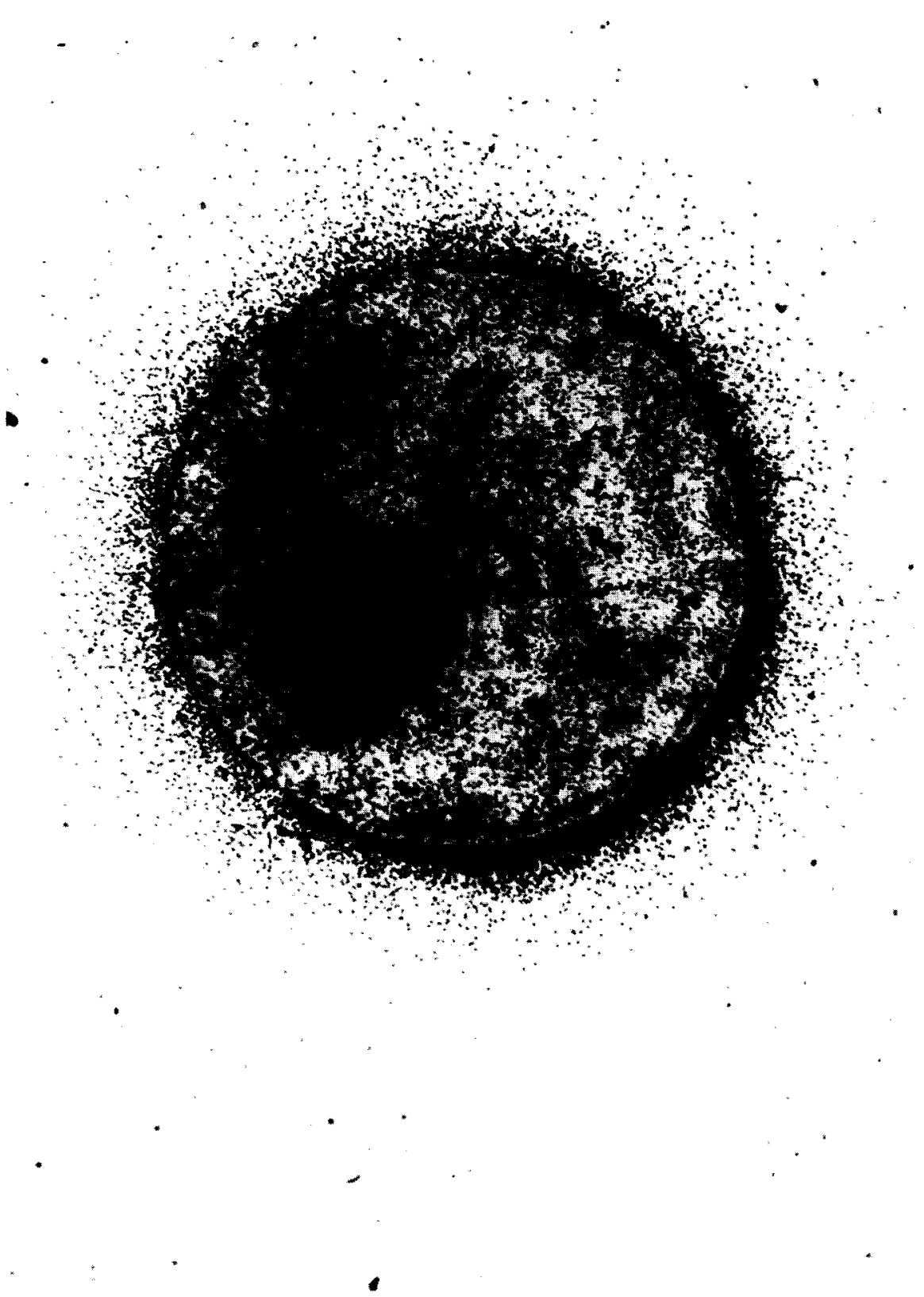

Fig. 2. Characteristic patterns of random human neutrophil migration under agarose after a $2 \mathrm{~h}$ incubation at $37^{\circ} \mathrm{C}$ observed at $15 \times$ magnification. A: random migration in $1 \%$ agarose containing $10 \%$ HINHS. B: random migration in $1 \%$ agarose containing $10 \%$ Sepharose-anti-trypsinized C5-absorbed HINHS. 


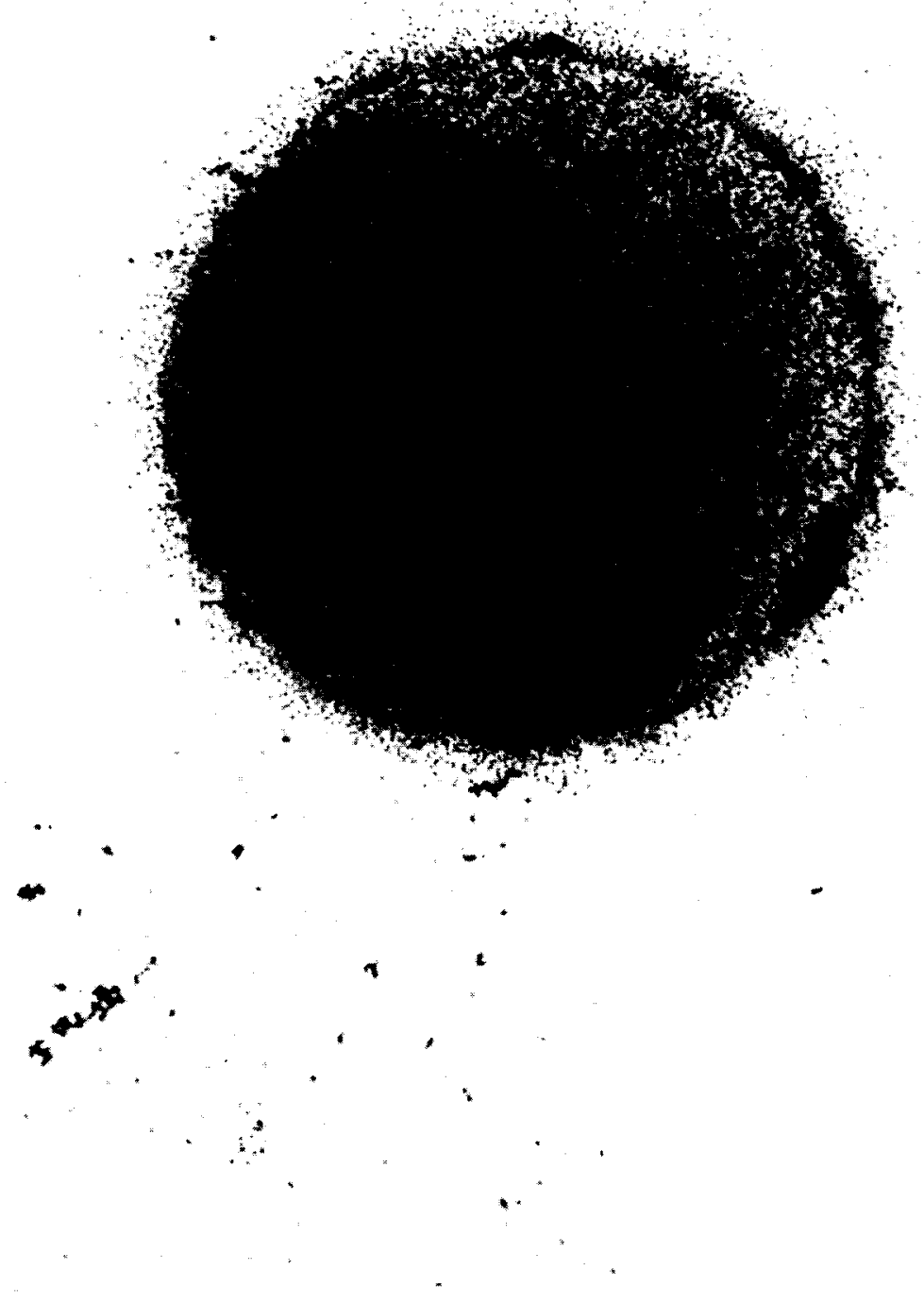

Fig. 2B. 

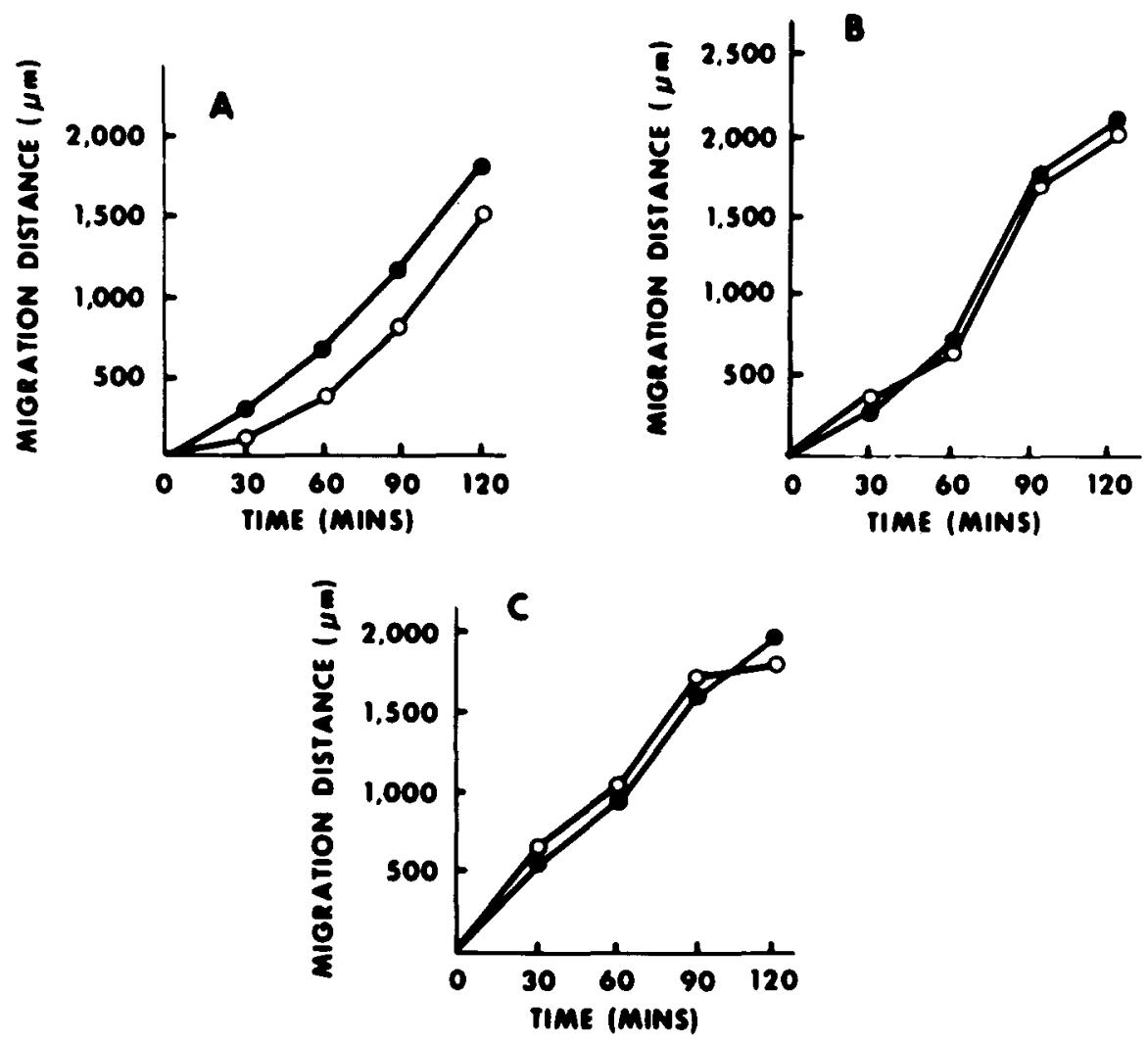

Fig. 3. Time-dependent neutrophil migration toward C5a des-arg or FMLP in $1 \%$ agarose containing either $10 \%$ HINHS or $10 \%$ anti-trypsinized C5-absorbed HINHS. A: migration toward C5a des-arg in agarose containing $10 \%$ HINHS $(-\bullet)$ or $10 \%$ S-anti-tryp-C5absorbed HINHS $(\bigcirc-O)$. Leukocytes were added to the central wells simultaneously with the application of C5a des-arg to the outer wells. B: same as A except FMLP was substituted for C5a des-arg. C: same as A except C5a des-arg was added $1 \mathrm{~h}$ prior to the application of leukocytes. Each data point represents the mean values of quadruplicate samples with S.D. $\leqslant 10 \%$ from a representative experiment.

enter the concentration gradient and move toward its source. Due to its larger size, C5a des-arg diffuses less rapidly in agarose than does FMLP (Repo and Kosunen, 1977). Since neutrophils randomly migrate faster in agarosecontaining HINHS (Fig. 1), they would presumably enter into the developing C5a des-arg gradient earlier than cells migrating in agarose-containing S-antitryp-C5-absorbed HINHS. This would explain the larger migration distances at all time intervals observed in the former (Fig. 3A).

The above experiments were repeated permitting $\mathrm{C} 5 \mathrm{a}$ des-arg to diffuse into the agarose for $1 \mathrm{~h}$ prior to the application of leukocytes. As shown in Fig. 3C, C5a des-arg-directed migration was now observed as early as $30 \mathrm{~min}$ after the application of cells in both. HINHS- and S-anti-tryp-C5-absorbed HINHS-containing agarose. More importantly, C5a des-arg-directed migration 
Fig. 4. Characteristic patterns of human neutrophil migration after $2 \mathrm{~h}$ incubation under agarose containing $10 \%$ HINHS or $10 \%$ anti-trypsinized C5-absorbed HINHS, visualized at $15 \times$ magnification. A: migration toward C5a des-arg (corresponds to the top of the photo) or E-MEM (corresponds to the bottom of the photo) in agarose containing $10 \%$ HINHS. B: same as A except 10\% S-anti-tryp-C5-absorbed HINHS was substituted for HINHS. C: migration toward FMLP (corresponds to the top of the photo) or E-MEM (corresponds to the bottom of the photo) in agarose containing $10 \%$ HINHS. D: same as C except $10 \%$ S-anti-tryp-C5-absorbed HINHS was substituted for HINHS. 
distances were approximately the same at each examined time interval in both systems. The migration patterns to C5a des-arg, FMLP, and the corresponding random migrations to E-MEM after $2 \mathrm{~h}$ incubation are shown in Fig. 4.

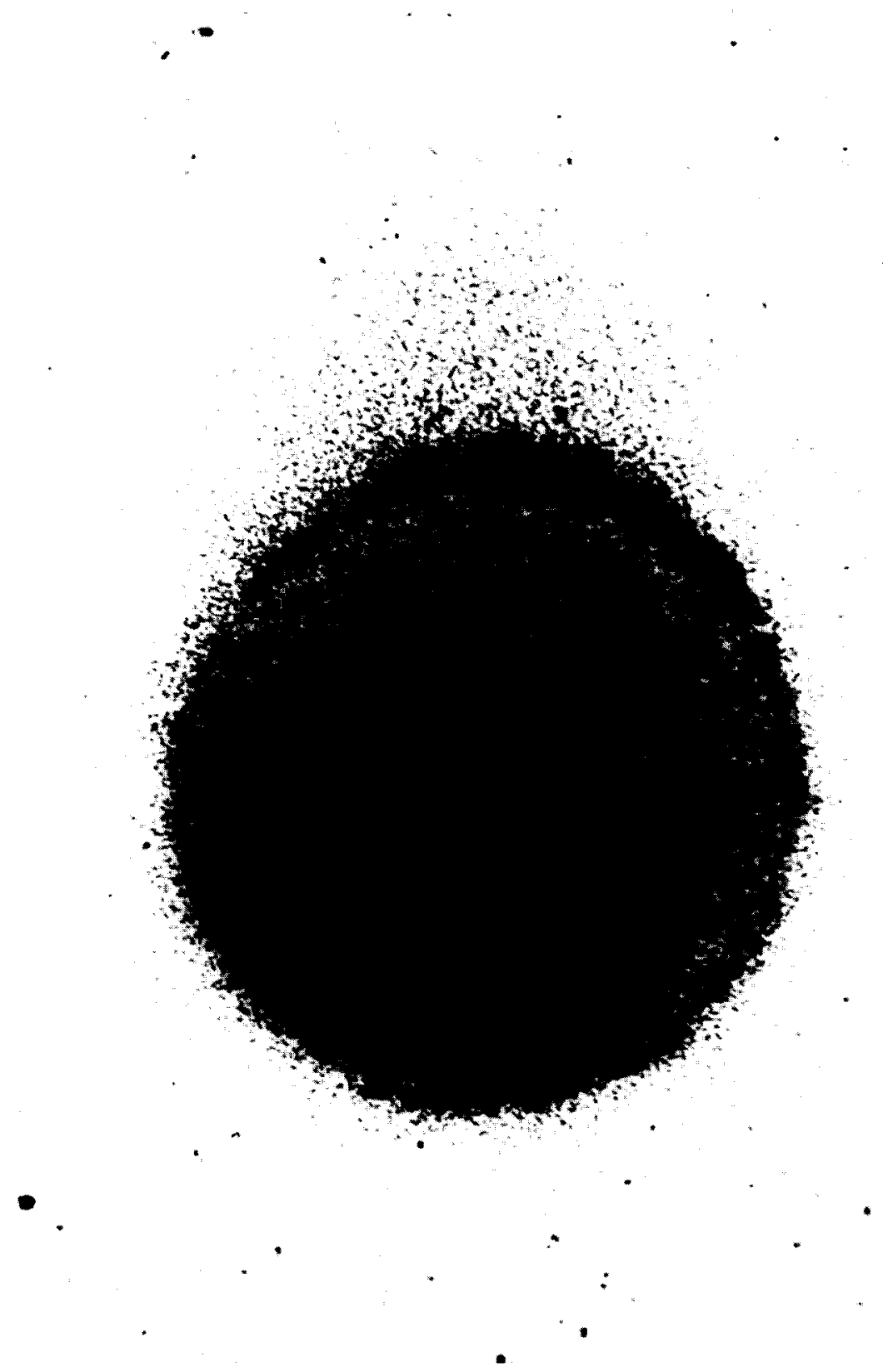

Fig. 4B. 
Comparison of C5a des-arg and FMLP chemotactic indices in agarose-containing HINHS or S-anti-tryp-C5-absorbed HINHS

Chemotactic indices (CI) were calculated by dividing the linear distances the neutrophils moved from the margin of the cell well toward either C5a

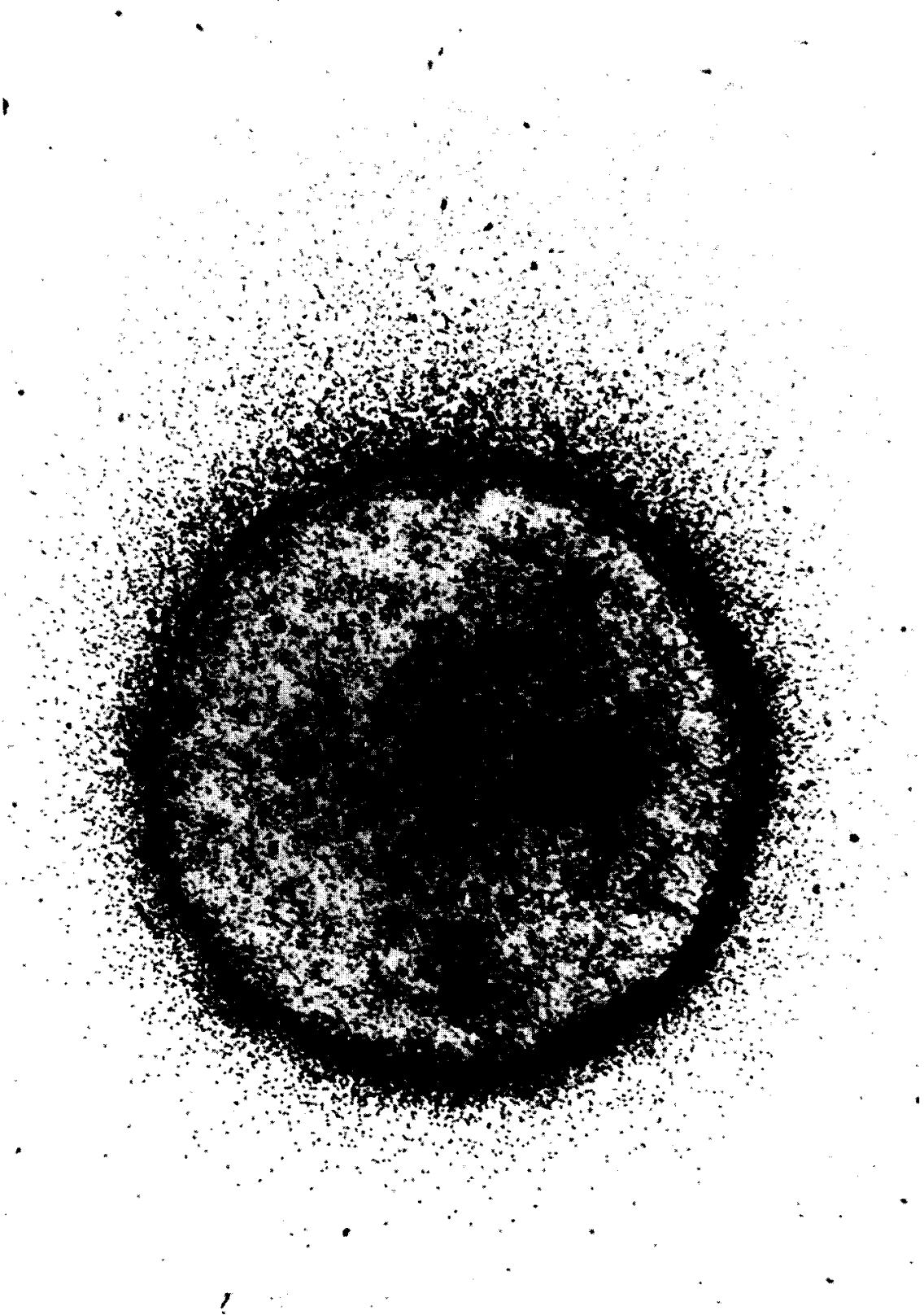

Fig. 4C. 
des-arg or FMLP by the random migration distances to E-MEM (Nelson et al., 1975). As shown in Fig. 5, CI to both C5a des-arg and FMLP were consistently higher in agarose containing S-anti-tryp-C5-absorbed HINHS. In the case of C5a des-arg (Fig. 5A, C), CI of up to 6.8 were achieved in the latter

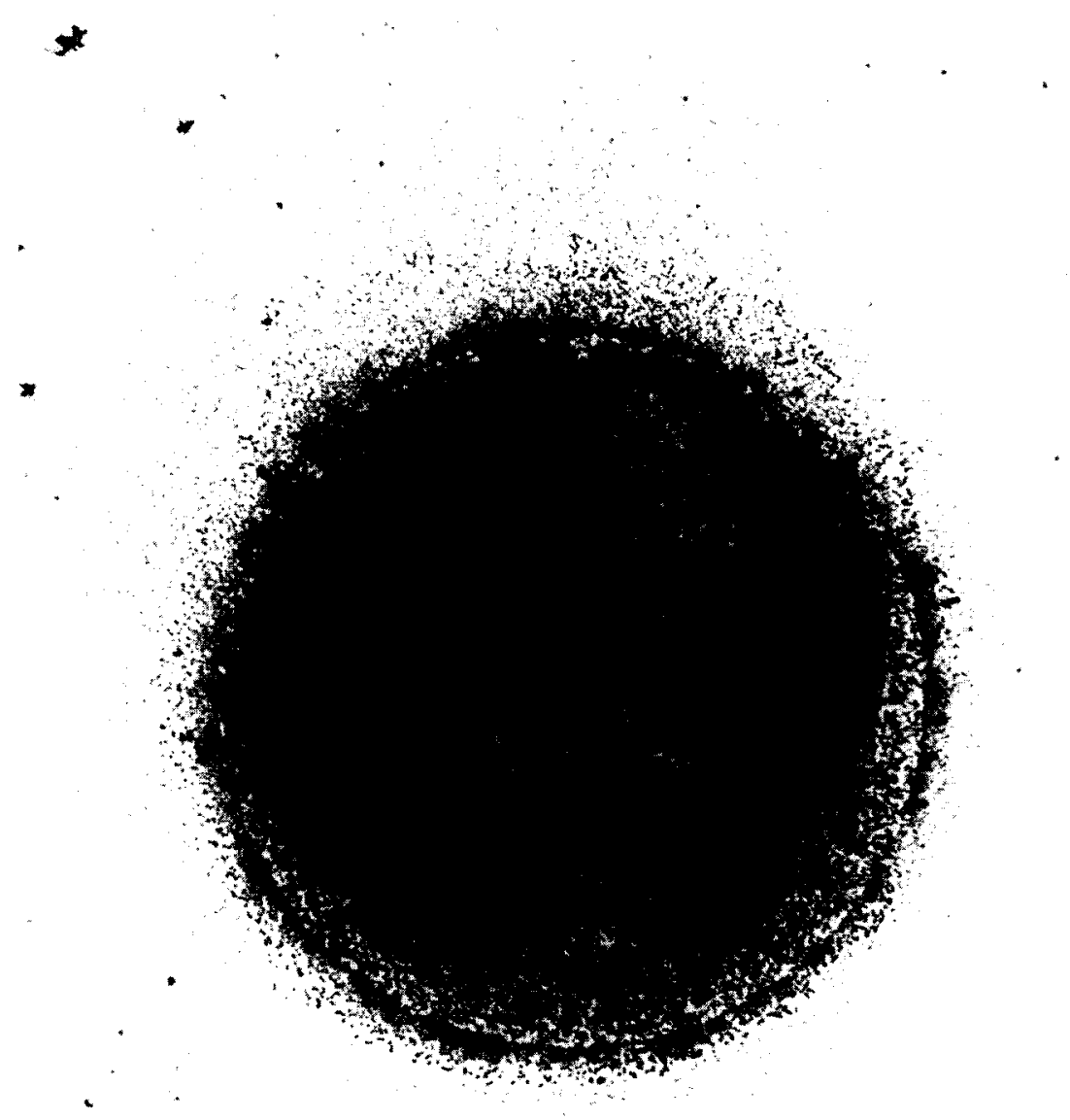

Fig. 4D. 

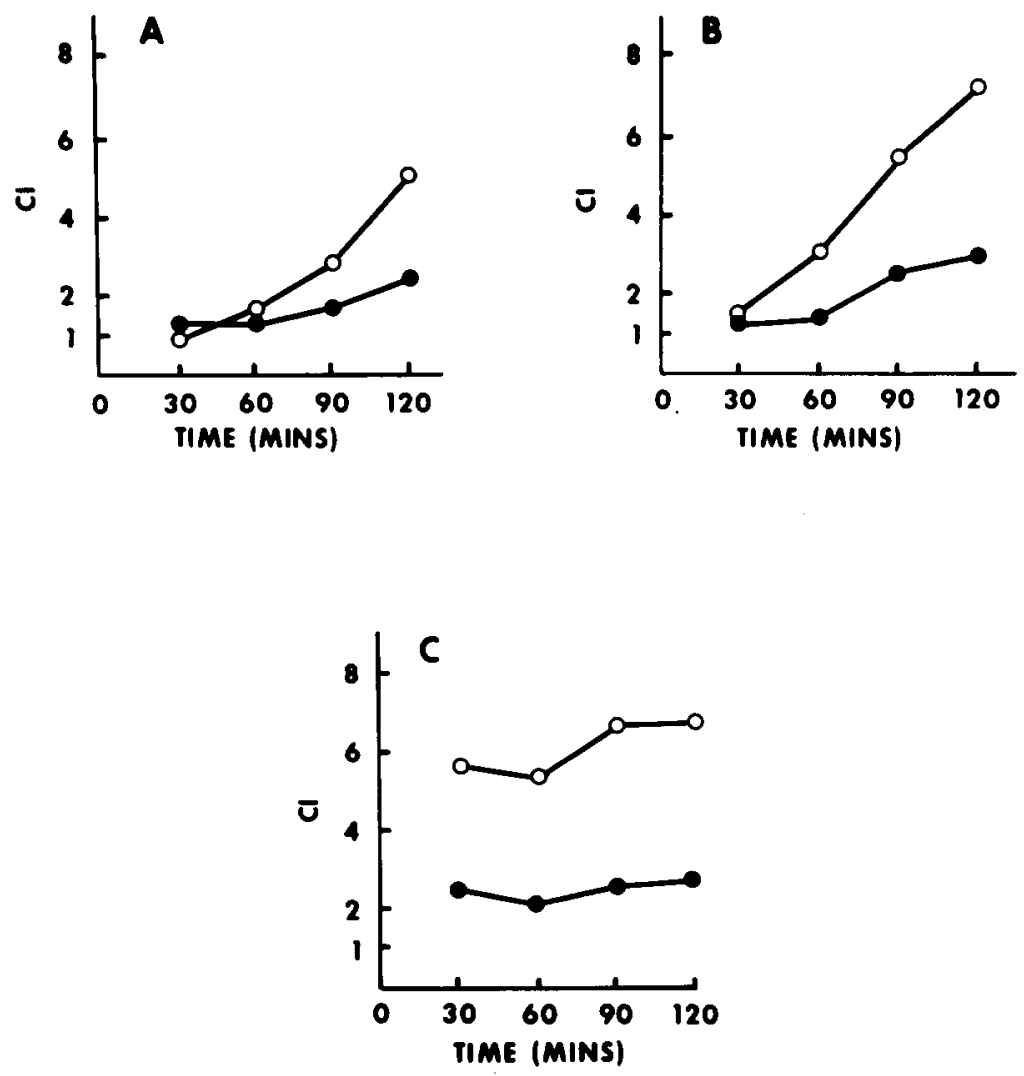

Fig. 5. Chemotactic indices as a function of time. A: C5a des-arg was added to the outer wells simultaneously with the application of leukocytes containing $2.5 \times 10^{5}$ neutrophils in $1 \%$ agarose plates containing $10 \%$ HINHS $(-0)$ or $10 \%$ Sepharose anti-trypsinized C5-absorbed HINHS (०-O). B: same as A except $10^{-7}$ M FMLP was substituted for C5a des-arg. C: same as A except C5a des-arg was added $1 \mathrm{~h}$ prior to the application of leukocytes. Each point represents mean values obtained from quadruplicate samples with S.D. $\leqslant 12 \%$.

system compared to maximal CI of 2.5 in agarose containing unabsorbed HINHS. Similar results were obtained when CI to FMLP were assessed (Fig. 5B). Maximum CI in agarose-containing HINHS or S-anti-tryp-C5-absorbed HINHS were approximately 3 and 7 , respectively.

Reduction of random neutrophil migration in agarose containing serum from different donor pools

Pooled normal human AB serum was obtained from a local blood bank, converted into serum, and heat-inactivated as described in Materials and Methods. Neutrophils were allowed to randomly migrate toward E-MEM for $2 \mathrm{~h}$ in $1 \%$ agarose containing $10 \%$ serum from each of 4 donor pools. As 
TABLE 2

Effect of absorption of pooled heat-inactivated human sera with Sepharose-anti-trypsinized $\mathrm{C5}$ on random human neutrophil migration under agarose $\mathrm{a}$.

\begin{tabular}{lll}
\hline $\begin{array}{l}\text { HINHS added } \\
\text { to agarose }\end{array}$ & Treatment & $\begin{array}{l}\text { Random migration (in } \mu \mathrm{m}, \text { mean } \pm \text { S.D. } \\
\text { from triplicate samples) }\end{array}$ \\
\hline Pool 1 & Unabsorbed & $420 \pm 70$ \\
& Absorbed & $170 \pm 30$ \\
Pool 2 & Unabsorbed & $810 \pm 50$ \\
& Absorbed & $220 \pm 20$ \\
Pool 3 & Unabsorbed & $580 \pm 55$ \\
& Absorbed & $150 \pm 30$ \\
Pool 4 & Unabsorbed & $510 \pm 60$ \\
& Absorbed & $250 \pm 35$ \\
\hline
\end{tabular}

a Ten microliters of E-MEM were added to both the inner and outer wells of $1 \%$ agarose plates containing $10 \%$ S-anti-tryp-C5-absorbed or unabsorbed HINHS. Leukocytes containing $2.5 \times 10^{5}$ neutrophils were added to the central wells and random migration assessed after $2 \mathrm{~h}$ incubation at $37^{\circ} \mathrm{C}$.

shown in Table 2, the random migration among these pools ranged from 430 to $810 \mu \mathrm{m}$.

The above experiment was repeated after absorbing serum from each of the 4 donor pools with S-anti-tryp-C5 prior to their incorporation into agarose. As shown in Table 2, random neutrophil migration was strikingly reduced and ranged from 150 to $250 \mu \mathrm{m}$ among the 4 pools.

\section{DISCUSSION}

Several cell types including polymorphonuclear leukocytes are capable of migrating spontaneously. Chemotactic factors are thought to influence this locomotion in at least two different ways. These factors may stimulate random migration, or if properly applied, directed migration (chemotaxis). The use of the migration under agarose method for the assessment of chemotaxis is based on the assumption that chemotactic factors diffuse from the attractant wells into the surrounding agarose. This results in the establishment of a chemotactic gradient extending from the attractant wells toward the wells containing cells. Leukocytes will migrate in such a milieu toward the source of attractant. One problem associated with the use of serum in the agarose system is that heat stable factors with chemotactic properties have been shown to occur in serum (Stetcher et al., 1971). The presence of such factors, uniformly distributed in agarose, can enhance random neutrophil migration.

In this report we describe a modification of the migration under agarose method which substitutes Sepharose-anti-trypsinized C5-absorbed HINHS 
for HINHS in agarose. This modification consistently reduced random neutrophil migration by at least $50 \%$ without significantly affecting C5a des-argor FMLP-directed migration. In addition, this modification diminished the range of random migration distances induced by different serum pools. A further advantage of this modification is its simplicity. Two hundred $\mathrm{ml}$ of Sepharose-anti-trypsinized C5 have been used to absorb up to 1 liter of human serum at a time. After use, the conjugate can be washed with $0.1 \mathrm{M}$ glycine $\mathrm{HCl}$ buffered saline ( $\mathrm{pH} 2.8$ ) and recycled repeatedly.

That Sepharose-anti-trypsinized C5 effectively absorbs C5-derived chemotactic activity from human serum is shown on Table 1. Following absorption with this conjugate, both HIZAS and C5a des-arg were no longer capable of inducing directed neutrophil migration. The absorbed chemotactic activity was recovered from the conjugate with $0.2 \mathrm{M}$ glycine $\mathrm{HCl}$ buffered saline and could subsequently be utilized in chemotactic assays. This implies that absorption from normal serum of C5-derived chemotactic peptides or other chemotactic factors cross-reacting antigenically with C5derived factors accounts for the substantial reduction in random neutrophil migration.

Taken together, C5a and C5a des-arg (the des-arg 74 derivative of C5a) are considered to be the most potent and physiologically important of the known chemotactic factors (Fernandez et al., 1978; Henson et al., 1978). Complement C5-dependent chemotactic activity may be generated by the action of neutrophil (Ward and Hill, 1970; Goldstein and Weissmann, 1974) and platelet (Weksler and Coupal, 1973) granule-associated enzymes. Mechanical forces generated during clot formation can damage these blood cells causing them to release their chemotactic factors into the serum.

Interactions between the complement system and coagulation pathways resulting in chemotactic activity have been documented. The action of thrombin on fibrinogen resulting in clot formation, for example, generates chemotactic activity (Kay et al., 1973). Activation of Hageman factor generates chemotactic activity by triggering intrinsic coagulation, fibrinolytic, and kinin generating pathways (Kay and Kaplan, 1975). It has recently been demonstrated that a $28,000 \mathrm{Mr}$ fragment from activated Hageman factor activates several complement components including C5 (Ghebrehiwet et al., 1981). Activated Hageman factor converts prekallikrein to kallikrein which, in turn, has been shown to cleave C5 and generate chemotactic peptides (Wiggins and Giclas, 1980). The chemotactic activity produced by these mechanisms under physiological conditions, however, is very low compared to that generated in serum by agents known to activate the classical or alternate complement pathways (Weiss et al., 1974). In support of this finding is the observation in the agarose system that HINHS exhibits very little chemotactic activity compared to HIZAS (Table 1; Nelson et al., 1975).

A major question raised by the data presented in this report is to what extent complement-derived chemotactic activity in HINHS is generated during the collection of serum or plasma and how much of it is present in the 
circulation of normal blood donors. Weisdorf et al. (1980) have found that C5a infused into rabbits is cleared from the vascular system exponentially with a half-life of $3 \mathrm{~min}$. There are several known physiological mechanisms which are capable of inactivating chemotactic factors. Normal human serum contains chemotactic factor inactivators which degrade both complementderived and bacterial chemoattractants (Berenberg and Ward, 1973; Till and Ward, 1975; Ward and Ozols, 1976). Neutrophil granule lysates contain proteases which also can inactivate chemotactic factors (Ward and Hill, 1970; Wright and Gallin, 1977). In addition, the myeloperoxidase-hydrogen peroxide halide system catalyzes the inactivation of these factors (Clark and Klebanoff, 1979). Recently Chenoweth and Hugli (1980) have presented evidence of receptors on the surface of neutrophils which specifically and irreversibly bind ${ }^{125}$ I-labeled C5a. These and other mechanisms might act to maintain negligible levels of C5-derived chemotactic factors in the circulation of healthy individuals. It is reasonable to assume, therefore, that some, if not most, of the C5-derived chemokinetic activity present in plasma or serum is generated during the collection of blood from donors. Whatever the source of the C5-derived activity present in HINHS, its removal with S-anti-tryp-C5 substantially reduces random neutrophil migration and allows greater reproducibility among serum pools used in chemotaxis under agarose assays.

\section{REFERENCES}

Berenberg, J.L. and P.A. Ward, 1973, J. Clin. Invest. 52, 1200.

Chenoweth, D.E. and T.E. Hugli, 1980, Mol. Immunol. 17, 151.

Chenoweth, D.E., J.G. Rowe and T.E. Hugli, 1979, J. Immunol. Methods 25, 337.

Clark, R.A. and S.J. Klebanoff, 1979, J. Clin. Invest. 64, 913.

Cuatrecasas, P., M. Wilcheck and C.B. Anfinsen, 1968, Proc. Natl. Acad. Sci. U.S.A. 61, 636.

Cutler, J.E., 1974, Proc. Soc. Exp. Biol. Med. 147, 471.

Fernandez, H.N., P.M. Henson, A. Otani and T.E. Hugli, 1978, J. Immunol. 120, 109.

Ghebrehiwet, B., M. Silverberg and A.P. Kaplan, 1981, J. Exp. Med. 153, 665.

Goldstein, I.M. and G. Weissmann, 1974, J. Immunol. 113, 1583.

Gray, G.D., M.M. Mickelson and J.A. Crim, 1969, Immunochemistry 6, 641.

Henson, P.M., B. Zanolari, N.A. Schwartzman and S.R. Hong, 1978, J. Immunol. 121, 851.

Kaplan, A.P., A.B. Kay and K.F. Austen, 1972, J. Exp. Med. 135, 81.

Kay, A.B. and A.P. Kaplan, 1975, Br. J. Haematol. 31, 417.

Kay, A.B., D.S. Pepper and M.R. Ewart, 1973, Nature New Biol. 243, 56.

Lee, S.K., J. Singh and R.B. Taylor, 1975, Eur. J. Immunol. 5, 259.

Nelson, R.D., P.G. Quie and R.L. Simmons, 1975, J. Immunol. 115, 1650.

Rabinovitch, M., M.J. Stefano and M.A. Dziezanowski, 1980, J. Reticuloendothel. Soc. $27,189$.

Repo, H., 1977, Scand. J. Immunol. 6, 203.

Repo, H. and T.U. Kosunen, 1977, Scand. J. Immunol. 6, 211.

Stetcher, V.J., E. Sorkin and G.B. Ryan, 1971, Nature New Biol. 233, 95.

Tannous, R. and N. Cavender, 1980, J. Immunol. Methods 36, 349.

Till, G. and P.A. Ward, 1975, J. Immunol. 114, 843. 
Vallota, E.H. and H.J. Müller-Eberhard, 1973, J. Exp. Med. 137, 1109.

Ward, P.A. and J.H. Hill, 1970, J. Immunol. 104, 535.

Ward, P.A. and L.J. Newman, 1969, J. Immunol. 102, 93.

Ward, P.A. and J. Ozols, 1976, J. Clin. Invest. 58, 123.

Weisdorf, D.J., D.E. Hammerschmidt, H.S. Jacob and P.R. Craddock, 1980, J. Immunol. $124,1544$.

Weiss, A.S., J.I. Gallin and A.P. Kaplan, 1974, J. Clin. Invest. 53, 622.

Weksler, B.B. and C.E. Coupal, 1973, J. Exp. Med. 137, 1419.

Wiggins, R.C. and P.C. Giclas, 1980, Fed. Proc. 39, 1049.

Wright, D.G. and J.I. Gallin, 1977, J. Immunol. 119, 1068. 